population. This was confirmed using a neutralising antibody to IL-17A, which resulted in a reduction in the size of ELS as determined by immunofluorescence detection of $\mathrm{T}$ and $\mathrm{B}$-cell involvement. The inhibition of ELS development by anti-IL17A treatment was also reflected by the reduced expression of lymphoid chemokines and AID. Notably, the infiltration of IL22-producing CD4 + cells, a key effector population involved in ELS formation, was also reduced in anti-IL-17-treated Il2 $7 \mathrm{ra}^{-/-}$but not WT mice.

Conclusions Here we show that IL-27 has a non-redundant inhibitory role in the regulation of the magnitude of ectopic germinal centre responses in inflamed SG. In the absence of a regulatory IL-27 signal, an exaggerated Th17 cell response was linked to dysregulated ELS size and activity. These findings provide new insights into the mechanisms governing ELS formation and highlight the role of IL-27 as an endogenous inhibitor of lymphoid neogenesis which could be exploited for therapeutic purposes in autoimmune diseases.

Disclosure of interest None declared

\section{P080 C-REACTIVE PROTEIN: NOT ONLY A MARKER, BUT ALSO A CAUSE OF INFLAMMATION THROUGH METABOLIC REPROGRAMMING OF HUMAN MACROPHAGES}

\footnotetext{
1,2 M Newling, 1,2L Sritharan, 1,2D Baeten, 1,2J Den Dunnen*. 'Department of Clinical Immunology and Rheumatology, Amsterdam Rheumatology and Immunology Centre, Academic Medical Centre, University of Amsterdam; ${ }^{2}$ Experimental Immunology, AMC, Amsterdam, Netherlands
}

\subsection{6/annrheumdis-2018-EWRR2018.97}

Introduction C-reactive protein (CRP) is an acute-phase protein produced in high quantities by the liver in response to infection and during chronic inflammatory disorders such as rheumatoid arthritis (RA). As a consequence, CRP is in widespread clinical use as a general marker of inflammation. Although CRP is known to facilitate clearance of cell debris by phagocytic cells by binding to its ligand phosphocholine on dead cells, it is still unclear whether CRP displays additional immunological functions.

Objectives Here, we set out to investigate whether CRP, which is present in high concentrations in synovial fluid of active RA patients, also plays a role in the orchestration of inflammation in the inflamed joint.

Methods Human macrophages were differentiated from blood monocytes of healthy volunteers, or sorted from synovial fluid of RA patients. Cells were stimulated with complexed CRP (cCRP) and/or ligands for Toll-like receptors (TLRs) and NODlike receptors (NLRs), mimicking the stimuli in the inflamed joint. Responsible signalling pathways were identified using small molecule inhibitors and RNA interference. Metabolic pathways were identified using specific inhibitors and the Seahorse metabolic analyzer.

Results Strikingly, we here provide evidence that CRP is not only a marker, but also a cause of inflammation by strongly amplifying the production of RA-associated pro-inflammatory cytokines. We show that complex formation of CRP as a result of binding to its ligand phosphocholine selectively enhanced TNF $\alpha$, IL-1 $\beta$, and IL-23 production by human macrophages. While c-CRP did not induce cytokine production individually, c-CRP synergized with TLRs and NLRs to amplify cytokine gene translation. We identified Fc gamma receptor I and IIa (FcyRI and FcyRIIa) as the main receptors responsible. Moreover, we unravelled the responsible molecular mechanism of c-CRP-induced inflammation, which crucially depends on signalling through kinases Syk and PI3K, resulting in enhanced gene translation of pro-inflammatory cytokines through metabolic reprogramming, particularly through amplified glycolysis and fatty acid synthesis.

Conclusions These data indicate that CRP is not only a marker, but also a cause of inflammation in RA patients by selectively promoting RA-associated pro-inflammatory cytokine production by human macrophages, thereby exacerbating pathology. From a therapeutic point of view, inhibition of c-CRP-induced immune activation, e.g. by targeting the identified molecular mechanisms, may be a valuable tool to suppress inflammation.

Disclosure of interest M. Newling: None declared, L. Sritharan: None declared, D. Baeten Employee of: Part-time employee UCB, J. Den Dunnen: None declared

\section{P081 SCLEROSTIN AFFECTS RANKL-MEDIATED OSTEOCLAST DIFFERENTIATION}

${ }^{1} \mathrm{~J}$ Intemann ${ }^{*},{ }^{2} \mathrm{C}$ Wehmeyer, ${ }^{1} \mathrm{~V}$ Kracke, ${ }^{1} \mathrm{E}$ Werbenko, ${ }^{1} \mathrm{P}$ Paruzel, ${ }^{3} \mathrm{I}$ Kramer, ${ }^{3} \mathrm{M}$ Kneissel, ${ }^{1} \mathrm{~T}$ Pap, ${ }^{1} \mathrm{~B}$ Dankbar. 'Institute of Musculoskeletal Medicine, University Hospital Muenster, Muenster, Germany; ${ }^{2}$ Institute of Inflammation and Ageing, University of Birmingham, Birmingham, UK; ${ }^{3}$ Novartis Institutes for BioMedical Research, Basel, Switzerland

\subsection{6/annrheumdis-2018-EWRR2018.98}

Introduction Sclerostin is a Wnt inhibitor and has anti-anabolic effects on bone formation by negatively regulating osteoblast differentiation. The lack of sclerostin in humans and mice leads to a higher bone mass and bone strength known as sclerosteosis. Therefore, inhibition of sclerostin is currently investigated as a treatment against osteoporosis. Surprisingly, the genetic deficiency or pharmacological inhibition of sclerostin causes a deterioration of disease severity in a TNF $\alpha$-dependent arthritis mouse model (hTNFtg). hTNFtg mice lacking sclerostin displayed enhanced joint inflammation, cartilage loss and bone erosion associated with an elevated number of osteoclasts within the joint.

Objectives We want to investigate the impact of sclerostin on osteoclast differentiation and bone erosion in arthritis.

Methods Sclerostin knockout ( sost $^{-/}$) mice were crossbred with hTNFtg mice to obtain sost ${ }^{-/} /$hTNFtg synovial fibroblasts. Co-cultures of synovial fibroblasts and wildtype bone marrow macrophages were analysed by TRAP staining. RANKL expression was measured by ELISA and cytokine expression by array analysis and Western Blot. Moreover, the influence of sclerostin on osteoclastogenesis was additionally analysed in mono-cultures.

Results In our co-culture system of synovial fibroblasts and bone marrow derived macrophages, fibroblasts from $\mathrm{sost}^{-/-}$ hTNFtg mice strongly promote osteoclastogenesis in comparison to hTNFtg synovial fibroblasts. Notably, no increased expression of receptor activator of NF-kB ligand (RANKL) was detectable in sost $^{-/} / \mathrm{hTNF}$ tg fibroblasts even after stimulation with inflammatory cytokines. Interestingly, basal secretion of IL-1 $\alpha$, which is known to stimulate osteoclastogenesis, was higher in sost ${ }^{-/} / \mathrm{hTNF}$ tg compared to hTNFtg fibroblasts. Accordingly, sclerostin inhibited osteoclastogenesis when administered in the pre-differentiation phase, whereas no effect was observed in the differentiation phase, indicating an inhibitory effect of sclerostin on osteoclast precursors.

Conclusions Sclerostin deficiency in hTNFtg synovial fibroblasts promotes RANKL-mediated osteoclastogenesis, which is most likely dependent on IL- $1 \alpha$.

Disclosure of interest None declared 